\title{
Reconstructing Digital Sets from X-rays
}

\author{
E. Barcucci ${ }^{1}$, A. Del Lungo ${ }^{1}$, M. Nivat ${ }^{2}$, R. Pinzani ${ }^{1}$, A. Zurli $^{1}$ \\ 1 Dipartimento di Sistemi e Informatica, Via Lombroso 6/17, Firenze, Italy, \\ pireoingfil.ing.unifi.it \\ 2 LITP Institut Blaise Pascal, Université Paris 7 "Denis Diderot", 2 Place Jussieu, \\ Paris Cedex 05, France, Nivat@litp.ibp.fr
}

\begin{abstract}
In this paper, we study the problem of determining digital sets by means of their X-rays. An X-ray of a digital set $F$ in a direction $u$ counts the number of points in $F$ on each line parallel to $u$. A class $\Phi$ of digital sets is characterized by the set $U$ of directions if among all $\Phi^{\prime}$ 's elements, each element in $\Phi$ is determined by its X-rays in $U$ 's directions. This discrete tomography's problem is of primary importance in reconstructing three-dimensional crystals from two-dimensional images taken by an electron microscope by measuring the number of atoms lying on each line in some directions (see [16]). There are some classes of digital sets that satisfy some connection and convexity conditions and that cannot be characterized by any set of directions [2]. Gardner and Gritzmann [10] show that there are some sets of four prescribed directions that characterize the class of totally convex sets (sets which are convex with respect to all the directions). We make the conjecture that there is a certain set $U$ of four directions that characterizes the class of convex polyominoes (sets which are convex with respect to only two directions: horizontal and vertical). In order to give experimental evidence of this conjecture, we present a polynomial algorithm that reconstructs convex polyominoes from their $\mathrm{X}$-rays.
\end{abstract}

\section{Introduction}

A digital set is a finite subset of the integer lattice $\mathbb{Z}^{2}$ defined up to a translation; it can be represented by a binary matrix or a set of cells (unitary squares), as shown in figure 1 . An $X$-ray of a digital set in a direction $u$ is a function giving the number of its points on each line parallel to $u$.

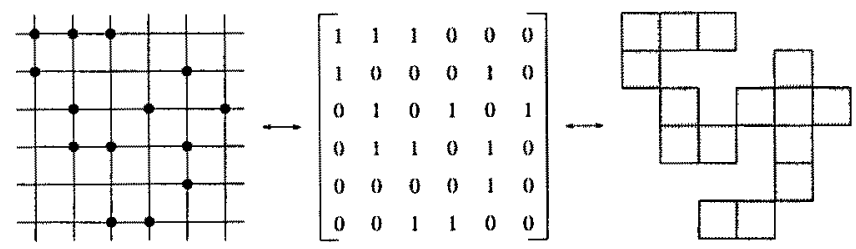

Fig. 1. Correspondence between a digital set, a binary matrix and a set of cells.

The reconstruction of a digital set from X-rays is of primary importance in computer-aided tomography, pattern recognition, image processing and data 
compression $[5,6,7,11,12,13,17]$. In detail, it can also be of help in reconstructing images taken by an electron microscope. During the symposium on Discrete Tomography: Algorithm and Complexity organized by G. Gritzmann and M. Nivat in Dagstuhl, Peter Schwander (a physicist at the Institute for semiconductor Physics in Frankfurt), had asked discrete tomography's experts for help in reconstructing three-dimensional crystals from two-dimensional projections taken by an electron microscope. He uses a new technique based on high resolution transmission electron microscopy (HRTEM) that can effectively measure the number of atoms lying on each line in some directions (see [16]).

In studying the problem of determining digital sets by $\mathrm{X}$-rays, many authors only use the horizontal and vertical ones $[1,4,5,7,8,12,14]$. Given a digital set $F$ and its matrix representation $A=\left(a_{i j}\right)$, the X-rays in the horizontal and vertical directions are defined as $H=\left(h_{1}, h_{2}, \ldots, h_{m}\right)$ and $V=\left(v_{1}, v_{2}, \ldots, v_{n}\right)$ respectively, where:

$$
h_{i}=\sum_{j=1}^{n} a_{i j} \quad 1 \leq i \leq m, \quad v_{j}=\sum_{i=1}^{m} a_{i j} \quad 1 \leq j \leq n .
$$

In this case, the number $h_{i}$ is the number of 1 in the $i$ th row, and $v_{j}$ is the number of 1 in the $j$ th column of $A$. The main problem met with in the reconstruction from horizontal and vertical X-rays is the "ambiguity" involved because, in some cases, a great many sets have the same pair $(H, V)$. The following two strategies have been adopted to reduce this ambiguity:

- more than two X-rays are assigned (see $[6,15]$ );

- some properties of the set to be reconstructed are given "a priori" (for example: convexity, connection and symmetry) and the algorithms take advantage of this further information to reconstruct the set (see $[1,5,8,12]$ ).

As far as the latter method is concerned, some properties imposed on the set completely eliminate the ambiguity, while some others only partially reduce it. It is shown in [9] that there is an exponential number of convex polyominoes having the same horizontal and vertical X-rays. Therefore, when convexity conditions are imposed on the sets, the ambiguity is usually reduced but not eliminated. We also wish to point out that some convexity conditions do not help us to reconstruct the digital set. For example, the problem of reconstructing a column-convex polyomino (i.e. a digital set which is convex with respect to only vertical direction) from horizontal and vertical $\mathrm{X}$-rays $(H, V)$ is $\mathrm{NP}$-complete [1]. In this paper, we begin by examining the problem of reconstructing a digital set from horizontal and vertical $\mathrm{X}$-rays $(H, V)$ and its extension to the problem of reconstructing a pattern from more than two X-rays' sets. We study the ambiguity problem with respect to some classes of digital sets on which some connection constraints are imposed. In particular, given a class $\Phi$ of digital sets, we want to know if a set $U$ of directions exists such that among all $\Phi$ 's elements, each element in $\Phi$ is determined by its X-rays in $U$ 's directions. If the set $U$ exists, we say that the class $\Phi$ is characterized by $U$. We then go on to establish the existence of a set of directions that completely eliminates ambiguity on $\Phi$. 
By extending the concept of switching component introduced by Chang and Ryser $[4,14]$, we can prove that there are some classes of digital sets that satisfy some connection and convexity conditions and that cannot be characterized by any set of directions. One of these classes is the set of column-convex polyominoes. We go on to study the ambiguity problem with respect to the class of convex polyominoes (i.e. digital sets which are convex with respect to only horizontal and vertical directions). We theorize that a certain set $U$ of four directions exists which allows us to eliminate ambiguity from convex polyominoes. We made an experimental verification of this conjecture that consists of two steps:

1. the uniform random generation of a convex polyomino $F$ having a specified number of rows and columns and the determination of $F$ 's X-rays in the four directions $U$;

2. the reconstruction of $F$ by means of the four discrete $\mathrm{X}$-rays determined in the previous step.

The second step is performed by means of an algorithm that starts out from the four X-rays obtained in the second step and reconstructs the convex polyominoes having these X-rays in $U$ 's directions. This algorithm takes advantage of the convexity properties and is very efficient. It is described in section 3 .

\section{Preliminaries}

A direction is an unit vector of the Euclidean bidimensional space $\mathbb{E}^{2}$. If $u$ is a direction, we denote the line through the origin parallel to $u$ by $l_{u}$. A discrete direction is a direction $u=\left(u_{x}, u_{y}\right)$ such that $\frac{u_{y}}{u_{x}}$ is a rational number. If $F$ is a digital set and $u$ is a discrete direction, then the discrete $X$-ray of $F$ in the direction $u$ is the function $X_{u} F$, defined as:

$$
X_{u} F(x)=\left|F \cap\left(x+l_{u}\right)\right|
$$

for $x=(n, m)$ with $n, m \in \mathbb{Z}$ (see figure 2).

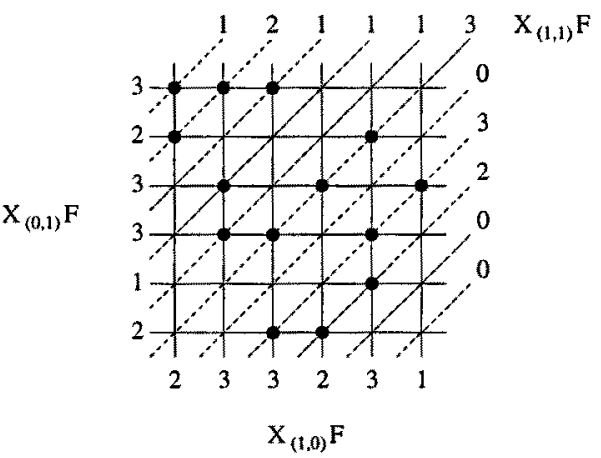

Fig. 2. Example of X-rays in the directions $u_{h}=(1,0), \quad u_{v}=(0,1), \quad u_{1}=(1,1)$. 
Let $U$ be a finite set of discrete directions and $\Phi$ be a class of digital sets. We say that $F \in \Phi$ is determined by the discrete X-rays in the directions of $U$ if whenever $F^{\prime} \in \Phi$ and $X_{u} F=X_{u} F^{\prime}$ for all $u \in U$, we have $F=F^{\prime}$. Otherwise, $F$ is ambiguous with respect to $U$ in $\Phi$. In both cases, we say that $F$ satisfies the set of X-rays $X_{u} F$. We say that the class $\Phi$ is characterized by the set $U$ of directions if each set in $\Phi$ is determined by the discrete $\mathrm{X}$-rays in the directions of $U$.

We can now define the following problem: "Given a class $\Phi$ of digital sets, is there a finite set $U$ of discrete directions characterizing $\Phi$ ?"

We approach this problem by defining some classes by means of the correspondence among the digital sets, the binary matrices and the sets of cells whose cardinality is unrestricted. A polyomino is a connected finite set of adjacent cells lying two by two along a side, and defined up to a translation (see figure 3 ).

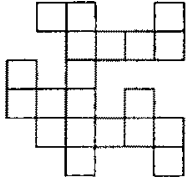

(a)

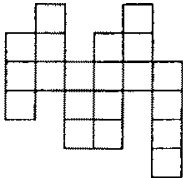

(b)

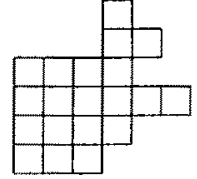

(c)

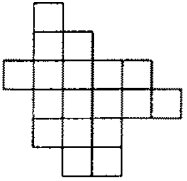

(d)

Fig. 3. A polyomino (a) and column-convex (b), row-convex (c), convex polyomino (d).

Let $F$ be a digital set. A column (row) of $F$ is the intersection of $F$ with a line $x=n(y=n), n \in \mathbb{Z}$. We say that a digital set $F$ verifies the properties $\mathbf{p}$, $\mathbf{v}$ and $\mathbf{h}$ if:

- p: $F$ is a polyomino;

- h: every row of $F$ is a connected set;

- v: every column of $F$ is a connected set.

A set $F$ belongs to the class $(\mathbf{x})(F \in(\mathbf{x}))$ if and only if it verifies the property $\mathbf{x}$. We denote the set of all the digital sets by $(\varnothing)$. We now introduce the following definitions:

- $F$ is a column-convex (row-convex) polyomino if $F \in(\mathbf{p}, \mathbf{v}), \quad(F \in(\mathbf{p}, \mathbf{h}))$,

- $F$ is a convex polyomino if $F \in(\mathrm{p}, \mathrm{h}, \mathrm{v})$.

We wish to point out that a convex polyomino can be considered to be the intersection between a set $P \in \mathbb{E}^{2}$, which is convex with respect to vertical and horizontal directions, and the lattice $\mathbb{Z}^{2}$.

In [2] we showed the following properties:

Theorem 1. There is not a finite set of discrete directions $U$ that characterizes the following classes: $(\varnothing),(\mathbf{p}),(\mathbf{v}),(\mathbf{h}),(\mathbf{p}, \mathbf{v}),(\mathbf{p}, \mathbf{h}),(\mathbf{h}, \mathbf{v})$.

Theorem 2 . The class $(\mathrm{p}, \mathrm{h}, \mathrm{v})$ cannot be characterized by any set of three discrete directions. 
Gardner and Gritzmann [10] proved that totally convex sets (i.e., which are convex with respect to all the directions) are characterized by any set $U$ of four discrete directions having cross ratio $\rho(U) \notin Q=\left\{\frac{4}{3}, \frac{3}{2}, 2,3,4\right\}$. Given a set $U=\left\{u_{1}, u_{2}, u_{3}, u_{4}\right\}$ of discrete directions, the cross ratio $\rho(U)$ is defined as $\rho(U)=\frac{\left(h_{3}-h_{1}\right)\left(h_{4}-h_{2}\right)}{\left(h_{3}-h_{2}\right)\left(h_{4}-h_{1}\right)}$, where $u_{i}=\left(u_{i x}, u_{i y}\right), h_{i}=\frac{u_{i y}}{u_{x x}}$, for $i=1,2,3,4$ and $h_{1}<h_{2}<h_{3}<h_{4}$. If $u_{i}=(0,1)$, then $\rho(U)=\frac{\left(h_{j}-h_{k}\right)}{\left(h_{j}-h_{l}\right)}$, where $j, k, l \neq i$. In [2] we showed that convex polyominoes are not characterized by $U=\left\{u_{h}, u_{v}, u_{3}, u_{4}\right\}$ when $p(U) \in Q$. Furthermore, for any set of three discrete directions $\left\{u_{h}, u_{v}, u_{3}\right\}$ there are 15 directions $u_{4}$ such that $\rho\left(\left\{u_{h}, u_{v}, u_{3}, u_{4}\right\}\right) \in Q$. These results can be easily extended to any set of directions $\left\{u_{1}, u_{2}, u_{3}, u_{4}\right\}$ and to the class of totally convex sets. On the basis of the previous results we make the following conjecture:

Conjecture 1 The class $(\mathrm{p}, \mathrm{h}, \mathrm{v})$ is characterized by $U=\left\{u_{h}, u_{v}, u_{3}, u_{4}\right\}$, where $\rho(U) \notin Q=\left\{\frac{4}{3}, \frac{3}{2}, 2,3,4\right\}$.

In order to obtain an experimental verification of our conjecture, we used an algorithm that reconstructs convex polyominoes from their discrete X-rays and considered the directions $U=\left\{u_{h}, u_{v},(2,1),(-1,2)\right\}$, which give the minimun size X-rays such that $\rho(U) \notin Q$. Our experiments consist of two steps:

- the uniform random generation of a convex polyomino $F$ having a specified number of rows and columns and the determination of $F$ 's X-rays in the four directions $U=\left\{u_{h}, u_{v},(2,1),(-1,2)\right\}$;

- the reconstruction of $F$ by means of the four discrete X-rays determined in the previous step.

The first step is carried out by using Polylab [3]. We obtain a random generation of four vectors that are consistent on the class $(\mathbf{p}, \mathbf{h}, \mathbf{v})$. The second step is performed by means of an algorithm that starts out from the four X-rays obtained in the first step and reconstructs the convex polyominoes having these X-rays in $U$ 's directions. If $U=\{(1,0),(0,1),(2,1),(-1,2)\}$ characterizes $(\mathbf{p}, \mathbf{h}, \mathbf{v})$, then, for each randomly generated convex polyomino $F$, the algorithm reconstructs $F$ from its X-rays in $U$ 's directions. In order to verify our conjecture experimentally, we verify that the algorithm only reconstructs the randomly generated convex polyomino $F$.

\section{Reconstruction algorithm}

Here we present a reconstruction algorithm derived from the one in [1]. Let us consider the X-rays along the set of directions $U=\left\{u_{h}, u_{v}, u_{3}, u_{4}\right\}$ with $u_{3}=(2,1)$ and $u_{4}=(-1,2)$. A convex polyomino $\Pi$ that satisfies them is contained in a rectangle $R$ of size $n \times m$, where $m=|H|$ and $n=|V|$. We call kernel any set $\alpha$ of cells such that $\alpha \subseteq \Pi \subseteq R$, and we call shell any set $\beta$ of cells such that $I \subseteq \beta \subseteq R$. Consequently, the shell contains $I$, while the kernel is contained in $\Pi$. Assuming that $\alpha=\emptyset$ and $\beta=R$, the basic idea of 
the algorithm that reconstructs $\Pi$ by starting out from the empty set $\emptyset$ is to reduce the shell and expand the kernel by means of some filling operations that take advantage of both the convexity constraint and the X-rays along $u_{h}, u_{v}$, $u_{3}, u_{4}$. The shell is reduced by eliminating the cells not belonging to $I I$ from $\beta$. Vice versa, the kernel is expanded by putting the cells belonging to $\Pi$ into $\alpha$. If $I I$ does not exist, the reconstruction fails, that is, the filling operations produce a kernel $\alpha$ and a shell $\beta$ such that $\alpha \not \subset \beta$. On the other hand, if only one polyomino $\Pi$ exists, that satisfies the given $\mathrm{X}$-rays, the reconstruction algorithm stops when $\alpha=\beta=\Pi$. However, if the quadruple $U=\left\{u_{h}, u_{v}, u_{3}, u_{4}\right\}$ does not characterize the class of convex polyominoes, it is likely that the reconstruction algorithm stops when $\alpha \subset \beta$ and $\alpha \neq \beta$, for a certain set of $\mathrm{X}$-rays along $U$. Consequently, $\Pi$ is indeterminate. In our tests, this never occurred. We use the following notations to describe the filling operations:

$-c(i, j)$ is the cell of $R$ 's in the $i$ th row and $j$ th column;

- $R^{i}$ is $R$ 's $i$ th row (the intersection of $R$ with the strip $\left.\mathrm{R} \times[i-1, i]\right)$;

- $R_{j}$ is $R$ 's $j$ th column (the intersection of $R$ with the strip $[j-1, j] \times \mathrm{R}$ );

- $\alpha^{i}, \beta^{i}$ are $\alpha$ 's $i$ th row $\left(\alpha^{i}=\alpha \cap R^{i}\right)$ and $\beta^{\prime}$ 's $i$ th row $\left(\beta^{i}=\beta \cap R^{i}\right)$ respectively;

- $\alpha_{j}, \beta_{j}$ are kernel $\alpha$ 's $j$ th column $\left(\alpha_{j}=\alpha \cap R_{j}\right)$ and shell $\beta$ 's $j$ th column $\left(\beta_{j}=\beta \cap R_{j}\right)$ respectively;

- $R_{i}^{(2,1)}, \alpha_{i}^{(2,1)}, \beta_{i}^{(2,1)}$ are $R$ 's, $\alpha$ 's and $\beta^{\prime}$ 's cells, respectively, that lie on the $i$ th parallel to the $(2,1)$ direction;

$-R_{i}^{(-1,2)}, \alpha_{i}^{(-1,2)}, \beta_{i}^{(-1,2)}$ are $R^{\prime} \mathrm{s}, \alpha$ 's and $\beta$ 's cells, respectively, that lie on the $i$ th parallel to the $(-1,2)$ direction;

- $h_{i}$ and $v_{j}$ are the $i$ th and the $j$ th components of the X-rays $X_{u_{h}} F(x)$ and $X_{u_{v}} F(x)$

- $p_{i}^{(2,1)}$ e $p_{i}^{(-1,2)}$ are the $i$ th component of the X-rays $X_{u_{1}} F(x)$ and $X_{u_{2}} F(x)$;

$-l\left(\alpha^{i}\right)=\min \left\{j \in[1 . . n]: c(i, j) \in \alpha^{i}\right\}, r\left(\alpha^{i}\right)=\max \left\{j \in[1 . . n]: c(i, j) \in \alpha^{i}\right\}$;

$-d\left(\alpha_{j}\right)=\min \left\{i \in[1 . . m]: c(i, j) \in \alpha_{j}\right\}, u\left(\alpha_{j}\right)=\max \left\{i \in[1 . . m]: c(i, j) \in \alpha_{j}\right\}$.

\section{Kernel expansion}

- Connecting operation $\Gamma$ on row $R^{i}$ :

1. if $\alpha^{i}=\varnothing$, then $\Gamma\left(\alpha^{i}\right)=\alpha^{i}$;

2. if $\alpha^{i} \neq \varnothing$, then $\Gamma\left(\alpha^{i}\right)=\left\{c(i, j): l\left(\alpha^{i}\right) \leq j \leq r\left(\alpha^{i}\right)\right\}$.

- Coherence operation $A$ on row $R^{i}$ :

1. if $\beta^{i}$ is disconnected, then $A\left(\alpha^{i}\right)=\alpha^{i}$;

2. if $\beta^{i}=\left\{c(i, j): j_{1} \leq j \leq j_{2}\right\}$ (that is, connected) and $\alpha^{i}=\varnothing$, then $\Lambda\left(\alpha^{i}\right)=\left\{c(i, j): j_{2}-h_{i}+1 \leq j \leq j_{1}+h_{i}-1\right\}$

3. if $\beta^{i}=\left\{c(i, j): j_{1} \leq j \leq j_{2}\right\}$ and $\alpha^{i} \neq \varnothing$, then $\Lambda\left(\alpha^{i}\right)=\alpha^{i} \cup\left\{c(i, j): l\left(\alpha^{i}\right) \leq j \leq j_{1}+h_{i}-1\right.$ or $\left.j_{2}-h_{i}+1 \leq j \leq r\left(\alpha^{i}\right)\right\}$.

- Coherence operation $A$ on cells $R_{i}^{(p, q)}$ for $(p, q)=(2,1),(p, q)=(-1,2)$ :

if the number of cells belonging to $\beta_{i}^{(p, q)}$ is equal to $p_{i}^{(p, q)}$, then $\Lambda\left(\alpha_{i}^{(p, q)}\right)=$ $\beta_{i}^{(p, q)}$. 


\section{Shell reduction}

- Connecting operation $\Phi$ on row $R^{i}$ :

1. if $\alpha^{i}=\varnothing$, then $\Phi\left(\beta^{i}\right)=\beta^{i}$;

2. if $\alpha^{i} \neq \varnothing$ and $c(i, k) \notin \beta^{i}$ and $k<l\left(\alpha^{i}\right)$, then $\Phi\left(\beta^{i}\right)=\beta^{i}-\{c(i, j): j \leq k\}$;

3. if $\alpha^{i} \neq \varnothing$ and $c(i, k) \notin \beta^{i}$ and $k>r\left(\alpha^{i}\right)$, then $\Phi\left(\beta^{i}\right)=\beta^{i}-\{c(i, j): j \geq k\}$.

- Coherence operation $\Theta$ on row $R^{i}$ :

1. if $\alpha^{i}=\emptyset$, then $\Theta\left(\beta^{i}\right)=\beta^{i}$;

2. if $\alpha^{i} \neq \emptyset$ then $\Theta\left(\beta^{i}\right)=\beta^{i}-\left\{c(i, j): j \leq r\left(\alpha^{i}\right)-h_{i}\right.$ or $\left.j \geq l\left(\alpha^{i}\right)+h_{i}\right\}$.

- Coherence operation $\Theta$ on cells $R_{i}^{(p, q)}$ for $(p, q)=(2,1),(p, q)=(-1,2)$ :

if the number of cells belonging to $\alpha_{i}^{(p, q)}$ is equal to $p_{i}^{(p, q)}$, then $\Theta\left(\beta_{i}^{(p, q)}\right)=$ $\alpha_{i}^{(p, q)}$.

As far as columns are concerned, connecting and coherence operations $\Gamma, \Lambda, \Phi, \Theta$ are defined in the same way as for the rows, by considering $\alpha_{j}, \beta_{j}, d\left(\alpha_{j}\right), u\left(\alpha_{j}\right), v_{j}$ instead of $\alpha^{i}, \beta^{i}, l\left(\alpha^{i}\right), r\left(\alpha^{i}\right), h_{i}$.

Assuming that a convex polyomino $\Pi$ exists that satisfies the quadruple of X-rays along $U$, kernel $\alpha$ and shell $\beta$ are two sets such that: $\alpha \subseteq \Pi \subseteq \beta$. In this situation, if we perform operations $\Gamma, A, \Phi$ and $\Theta$ on $R^{i}$ and $R_{j}$, we can easily prove that:

$$
\begin{array}{llll}
\alpha^{i} \subseteq \Gamma\left(\alpha^{i}\right) \subseteq \Pi^{i} & \alpha^{i} \subseteq \Lambda\left(\alpha^{i}\right) \subseteq \Pi^{i} & \Pi^{i} \subseteq \Phi\left(\beta^{i}\right) \subseteq \beta^{i} & \Pi^{i} \subseteq \Theta\left(\beta^{i}\right) \subseteq \beta^{i} \\
\alpha_{j} \subseteq \Gamma\left(\alpha_{j}\right) \subseteq \Pi_{j} & \alpha_{j} \subseteq \Lambda\left(\alpha_{j}\right) \subseteq \Pi_{j} & \Pi_{j} \subseteq \Phi\left(\beta_{j}\right) \subseteq \beta_{j} & \Pi_{j} \subseteq \Theta\left(\beta_{j}\right) \subseteq \beta_{j}
\end{array}
$$

Consequently, operations $\Gamma$ and $\Lambda$ expand $\alpha$, while $\Phi$ and $\Theta$ reduce $\beta$. It follows that the algorithm that reconstructs $I$ initially sets $\alpha=\emptyset, \beta=R$ and performs the filling operations on $R$ 's rows and columns in $\Gamma, \Lambda, \Phi$ and $\Theta$ order and sets $\alpha=\Gamma(\alpha), \beta=\Phi(\beta), \alpha=\Lambda(\alpha)$ and $\beta=\Theta(\beta)$ at each row or column.

\section{Conclusions}

As stated in section 2, we verify our conjecture experimentally in two steps:

1. the random generation of four vectors that are consistent on $(\mathbf{p}, \mathbf{h}, \mathbf{v})$;

2. the execution of the algorithm performing on the vectors of step (1).

We ran the algorithm up to thousands of cases and the algorithm always reconstructed one convex polyomino (the one randomly generated at step (1)) without any ambiguity. The results of these tests gave us an experimental evidence of both our conjecture and the good performances of the reconstruction algorithm. 


\section{References}

1. E. Barcucci, A. Del Lungo, M. Nivat and R. Pinzani, Reconstructing convex polyominoes from horizontal and vertical projections, Theor.Comp. Sci.155 (1996) 321347.

2. E. Barcucci, A. Del Lungo, M. Nivat, R. Pinzani, X-rays characterizing some classes of digital pictures, Research report RT 4/96, Dipartimento di Sistemi e Informatica, Università di Firenze (1996).

3. E. Bertoli, A. Del Lungo and F. Ulivi, POLYLAB, A package for the study of polyominoes, Proceedings of the 7th FPSAC, eds. B. Leclerc and J. Y. Thibon, Marne-la-Vallé, (1995) 65-74.

4. S. K. Chang, The reconstruction of binary patterns from their projections, Comm. ACM 14 (1971) 21-25.

5. S. K. Chang and C. K. Chow, The reconstruction of three-dimensional objects from two orthogonal projections and its application to cardiac cineangiography, IEEE Trans. Comput. C-22 (1973) 661-670.

6. S. K. Chang and G. L. Shelthon, Two algorithms for multiple-view binary pattern reconstruction, IEEE Trans. Syst., Man., Cybern. SMC-1 (1971) 90-94.

7. S. K. Chang and Y. R. Wang, Three-dimensional objects reconstruction from two orthogonal projections, Pattern Recognition 7 (1975) 167-176.

8. A. Del Lungo, Polyominoes defined by two vectors, Theor. Comp. Sci. 127 (1994) 187-198.

9. A. Del Lungo, M. Nivat and R. Pinzani, The number of convex polyominoes reconstructible from their orthogonal projections, Disc, Math 157 (1996) 65-78.

10. R. J. Gardner and P. Gritzmann, Discrete tomography: determination of finite sets by X-rays, to appear in Transactions of the AMS.

11. R. Gordon and G. T. Herman, Reconstruction of pictures from their projections, Graphics and Image Processing 14 (1971) 759-768.

12. A. Kuba, The reconstruction of two-directionally connected binary patterns from their two projections, Comp. Vision, Graphics, and Image Proc. 27 (1984) 249-265.

13. D. G. W. Omnasch and P. H. Heintzen, A new approch for the reconstruction of the right or left ventricular form from biplane angiocardiographic recordings, Digital Imaging in Cardiovascular Radiology, Georg Thieme Verlag, Stuttgart-New York, 1983, 151-163.

14. H. Ryser, Combinatorial Mathematics, The Carus Mathematical Monographs No. 14, The Mathematical Association of America, Rahway 1963.

15. A. R. Shliferstein and Y. T. Chien, Switching components and the ambiguity problem in the reconstruction of pictures from their projections, Pattern Recognition $10(1978) 327-340$.

16. P. Schwander, C. Kisielowski, M. Seibt, F. H. Baumann, Y. Kim and A. Ourmazd, Mapping projected potential, interfacial roughness, and composition in general crystalline solids by quantitative transmission electron microscopy, Physical Review Letters 71 (1993) 4150-4153.

17. C. H. Slump and J. J. Gerbrands, A network flow approach to reconstruction of the left ventricle from two projections, Comp. Graphics Image Proc.18 (1982) 18-36. 